\title{
Las revistas argentinas de filología, literatura y lingüística: visibilidad en bases de datos internacionales
}

\begin{abstract}
Susana Romanos de Tiratel
Directora del Instituto de Investigaciones Bibliotecológicas, Facultad de Filosofía y Letras U niversidad de Buenos Aires. Directora de la revista Información, Cultura y Sociedad. Profesora Titular del Área de Recursos y Servicios de Información del Departamento de Bibliotecología y Ciencia de la Información, Facultad de Filosofía y Letras U niversidad de Buenos Aires.

Correo electrónico: sromanos@filo.uba.ar
\end{abstract}

\section{G raciela M. Giunti}

Licenciada en Bibliotecología y Documentación, U niversidad de Buenos Aires. Secretaria Académica del Instituto de Investigaciones Bibliotecológicas, Facultad de Filosofía y Letras U niversidad de Buenos Aires. M iembro del Comité de Redacción de la revista Información, Cultura y Sociedad.

Correo electrónico: inibi@filo.uba.ar

\section{Alejandro E. Parada \\ Investigador del Instituto de Investigaciones Bibliotecológicas, Facultad de Filosofía y Letras U niversidad de Buenos Aires. Secretario de Redacción de la revista Información, Cultura y Sociedad. Jefe de la Biblioteca de la Academia Argentina de Letras. \\ Correo electrónico: aparada@filo.uba.ar}

\section{Resumen}

El artículo proporciona una introducción al mundo de la publicación periódica y a su problemática así como una breve historia de las revistas literarias argentinas. Dentro del marco teórico provisto por Kereztesi respecto de las interrelaciones entre investigación y bibliografía, se expone la metodología y los procedimientos adoptados para determinar los indicadores de representatividad, de visibilidad y de vacancia de las revistas argentinas de Filología, Literatura y Lingüística en bases de datos internacionales y latinoamericanas pluridisciplinarias e

internacionales unidisciplinarias. Finalmente, se discuten ciertos aspectos que se derivan del estudio realizado.

Palabras clave

Revistas argentinas; Filologia; Literatura; Lingüística; Indicadores de porcentaje y representatividad.

Argentinean journals of philology, literature and linguistics: visibility in international databases

\footnotetext{
Abstract

The article introduces periodical publication and addresses the questions raised in connection with this format, as well as a brief history of literary journals in Argentina. Within Kereztesi's framework of reference as regards the research/bibliography interface, the discussion focuses on the methods and procedures to determine indicators of representativity, visibility and vacancy of Argentinean journals in Philology, Linguistics and Literature. The research examines data available in both singlediscipline and multidisciplinary international and Latin-American bases. Matters arising as a result of our study are discussed in our Conclusion.
}

Keywords

Argentinean journals; Philology; Literature; Linguistics; Indicators of percentage and representativity.

\section{INTRODUCCIÓN}

El mundo de la revista especializada involucra una serie de actores que se conjugan para hacerla posible: autores; editores científicos; evaluadores; publicadores privados o institucionales; bibliógrafos que controlan los contenidos a través de la indización/ condensación, clasificación, etc. constituyendo bases de datos y generando servicios de acceso a los contenidos de las publicaciones periódicas; bibliotecólogos que seleccionan, adquieren, procesan, almacen an y conservan en sus colecciones los títulos y las bases de datos que permiten la recuperación de los artículos; y usuarios 0 consumidores de la producción científica. Las mismas personas pueden desempeñar roles distintos en diferentes momentos o simultáneamente.

Por otra parte, la revista especializada ha sido caracterizada como un canal de comunicación formal, público y ordenado al que se le reconocen tres funciones básicas: registro oficial de la ciencia, medio para difundir información e institución social que confiere prestigio y recompensas (Romanos de Tiratel, 2000: 182-83). Sin embargo, este canal de comunicación formal preferido, y en muchos casos excluyente, para comunicar los avances del conocimiento en todas las disciplinas no ha dejado de presentar conflictos. A continuación se resumirán algunos de sus principales problemas.

En primer término se ubica la proliferación de publicaciones periódicas causada por el incremento de las actividades de investigación y desarrollo, por el aumento del número de personas que publican, por la importancia acordada a la publicación como una medida de la estatura del científico por parte de sus pares y empleadores (síndrome del publish-or-perish), por la creciente especialización y compartimentación de las disciplinas, y por los desarrollos en la tecnología de impresión a alta velocidad; por otra parte, la proliferación no está dada sólo por la aparición de nuevos títulos sino también por su separación en varias secciones que, eventualmente, se vuelven revistas separadas. 


\section{Susana Romanos de Tiratel / Graciela M. Giunti / Alejandro E. Parada}

Directamente relacionado con lo antedicho está el fenómeno de la dispersión de trabajos sobre un tema dado en un gran número de revistas. Los estudios de citas han establecido que, en cualquier tópico, una proporción sustancial de los artículos se concentran en un número relativamente pequeño de títulos, y que los restantes están dispersos en un gran número de revistas periféricas 0 ajenas al tema (Ley de dispersión de Bradford). En cierto grado, este fenómeno puede ser conveniente para promover la fertilización cruzada de ideas y los descubrimientos azarosos, pero sus desventajas son mayores que las ventajas. Los dos problemas, el de proliferación de revistas y el de dispersión de artículos envuelven a autores, bibliotecólogos, productores de servicios secundarios, y usuarios de la literatura especializada.

0 tro aspecto a considerar es la demora en la publicación ocasionada por los procesos editoriales y evaluativos elaborados que, muchas veces, involucran revisiones amplias y repetidas del manuscrito; así, el tiempo que transcurre entre la remisión del primer manuscrito y su eventual aparición puede variar desde seis meses a dos años. Las demoras también pueden ser el resultado del envío del trabajo a varias periódicas hasta que se acepta y publica. Debido a la tardanza inherente al proceso de publicación en revistas, los investigadores recurren a una variedad de canales alternativos para difundir los resultados de su investigación: distribución de preimpresos por medios electrónicos, congresos y conferencias y, ocasionalmente, medios masivos.

U na cuestión fundamental es la diversidad de roles muchas veces incompatibles- que intenta desempeñar simultáneamente la revista científica, presentándose como un envoltorio muy general que ha tratado de ser demasiado para todo el mundo. Por ejemplo, el rol de registro de archivo necesita pasar - y esto con sume tiempo - a través de los procesos de edición y arbitraje, así, se entra en colisión con el rol de actualización permanente que requiere una publicación rápida, lo cual puede inhibir la edición y la evaluación elaboradas.

La caracterización unívoca de los títulos no es un problema menor dada la complejidad de algunos y la similitud o igualdad de otros en diferentes países. En parte, la solución ha sido proporcionada por el International Standard Serial Number (ISSN), código irrepetible e incompartible que, desde 1973, se le otorga a cada nueva revista o a una ya existente que ha tenido un cambio significativo en su título. El sistema funciona por medio de un centro internacional con sede en París que asigna bloques de códigos a los centros nacionales para que estos, a su vez, los otorguen a las revistas de sus respectivos países. El sistema se ha extendido con bastante rapidez $\mathrm{y}$, actualmente, son los mismos editores quienes solicitan el ISSN al organismo nacional correspondiente. Por supuesto, dado que el sistema no es obligatorio, de hecho, siguen existien do publicaciones especializadas sin ISSN, sencillamente, porque no se lo ha tramitado o porque cambian su título y no gestionan una nueva adjudicación del código. Además, y a pesar de los treinta años transcurridos desde su implantación, los servicios de indización y condensación no han adoptado una norma uniforme en cuanto a la obligatoriedad de su inclusión lo cual dificulta, en gran medida, la identificación de los títulos que analizan.

Por último, pero no por eso de menor importancia, hay que mencionar los costos crecientes de las revistas especializadas, lo que seha dado en Ilamar "journal crisis". $\mathrm{H}$ an sentido el impacto tanto los editores científicos como los investigadores y los bibliotecólogos. Las instituciones de investigación intentan resolver esta situación de diversas maneras: transferir el presupuesto para libros a la compra de publicaciones periódicas (que no es la política más conveniente para las disciplinas de H umanidades), adquirir cooperativamente, participar en redes o consorcios para compartir recursos y, cuando es in evitable, cancelar suscripciones. D en tro de este cuadro hay que diferenciar entre los títulos producidos por la mayoría de las sociedades científicas y universidades que, relativamente, tienen un costo más bajo y una alta calidad y los publicados comercialmente que, en promedio, son al rededor de cuatro veces más caros (Frazier, 2000: 119).

Esta apretadísima síntesis (H oughton, 1975; Day, 1976; Kronick, 1976; Piternick, 1989; Puccio, 1989; Licea de A renas, 1994; M orris, 1998; Nisonger, 1998) tiene el propósito de fundamentar la complejidad del tema, la diversidad de enfoques y la variedad de intereses asociados con la revista especializada.

De esta situación se deriva uno de los inconvenientes para procesar la bibliografía latinoamericana referida a la problemática que, a las cuestiones antes mencionadas, agrega otras propias de la región: falta de regularidad de las publicaciones periódicas atribuible a la escasez de fondos permanentes dedicados a su financiamiento, al amateurismo de los responsables de su concreción, a la carencia de políticas científicas claras y sostenidas en el tiempo, a fallas en la distribución y difusión de los títulos, etc.; presencia casi nula de títulos latinoamericanos en las bases de datos internacionales, sobre todo en las del Institute for Scientific Information; debate sobre el uso del idioma español o portugués; establecimiento de 
parámetros válidos y aceptados por todos para evaluar la calidad de las publicaciones; problema del número elevado de títulos en cada especialidad; oscilación continua entre dependencia e independencia de los países centrales o difusión endogámica de los resultados de investigación (C etto y Hillerud, comp., 1995; Fuenmayor P., 1995; Krauskopf y V era, 1995; A rmendáriz Sánchez y O rdóñez Alonso, 1998; C etto y A lonso, 1999; Taller de 0 btención de indicadores bibliométricos y de actividad científica, 2000 y 2003; G raças Targino y Garcia, 2000; H aupt, 2000; Ratto de Sala y Dellamea, 2001)

Los autores de los trabajos sobre estos temas juegan roles diversos en el ciclo de transferencia de la información (pueden ser productores, editores científicos, evaluadores, publicadores, funcionarios administrativos de centros de investigación, bibliógrafos, bibliotecólogos 0 consumidores) y, por lo tanto, persiguen objetivos que, la mayoría de las veces, colisionan entre sí o abordan las cuestiones desde la propia experiencia o la apreciación personal. Esto no significa desvalorizar esos aportes sino, más bien, ubicarlos dentro de una perspectiva más ajustada.

Por eso es importante contextualizar a este estudio dentro de la Bibliografía porque nace y se desarrolla a partir de la preocupación respecto de la falta de control bibliográfico de la producción argentina en Humanidades y en Ciencias Sociales ${ }^{(1)}$, y este artículo, en particular, se centra en las revistas especializadas en Filología, Literatura y Lingüística.

Un presupuesto subyacente es que "...compete a cada uno de los países el control exhaustivo de la literatura derivada de las actividades de investigación constituyéndose así la bibliografía nacional especializada de cada país..." (Romanos de Tiratel, 2000: 1). No es esta una presunción antojadiza sino que reconoce su origen en la historia misma de las publicaciones científicas en los países más desarrollados dado que, en 1665 y con una diferencia de tres meses, aparecen las dos primeras revistas eruditas - Journal des Sçavants y Philosophical transactions- que instauran un modelo de comunicación seguido por el resto de Europa. Poco más de cien años después (1778) y ante la necesidad de alertar sobre los avances y descubrimientos de sus pares nacionales y extranjeros se compila en Alemania la primera revista de resúmenes especializada, obra de Lorenz von C rell, que se tradujo al inglés en 1791. M uy pronto, esta tarea se presenta como imposible para un solo individuo y son las sociedades profesionales y las academias quienes asumen el proceso de organización y difusión considerándolo como uno de sus deberes básicos (H oughton, 1975: 81-82).

Sin embargo, este proceso no sigue las mismas etapas en la Argentina, donde sólo se registra un esfuerzo sostenido que cubrió la producción en Artes y Literatura entre 1959 y 1971 bajo la dirección de Augusto Raúl Cortazar y con el apoyo del Fondo $\mathrm{N}$ acional de las Artes ${ }^{(2)}$.

Ahora bien, se puede suponer que los servicios de indización y resúmenes de alcance internacional controlan, analizan y difunden la producción total de los países que abarcan y, por lo tanto, no es necesario que en cada lugar se hagan índices nacionales. C onfirmar o no este supuesto con datos fiables es uno de los objetivos perseguidos por este estudio que, en primer lugar, determinó los porcentajes de visibilidad de las revistas argentinas especializadas en $\mathrm{H}$ umanidades y Ciencias Sociales en bases de datos internacionales pluridisciplinarias (Romanos de Tiratel, 2002; Romanos de Tiratel, Giunti y Parada, 2002) y que ahora ha empezado a tomar cada materia, replicando el modelo ya probado, en las bases de datos internacionales unidisciplinarias.

En este caso, los resultados que se presentan tienen dos límites: uno disciplinar porque sólo se estudia la Filología, la Literatura y la Lingüística y el otro documental porque únicamente indaga qué revistas argentinas de esas áreas temáticas están indexadas en bases de datos internacionales, tanto pluridisciplinarias como unidisciplinarias.

\section{LAS REVISTAS LITERARIAS ARGENTINAS: BREVE ESBOZO HISTÓRICO}

La historia de las revistas literarias en la Argentina se caracteriza, inequívocamente, por las vicisitudes políticas y sociales que influyeron en la historia cultural del país durante el siglo xIx y xx. M uchos factores coadyuvaron en la aparición, más o menos orgánica, de esta clase de literatura periódica, tales como la organización nacional luego de Caseros (1852), la aparición de la pujante generación de 1880, la inmigración masiva, el extraordinario proceso de urbanización, y el desarrollo de la alfabetización pública y laica. H echos estos que terminaron por definir e identificar no sólo al escritor dentro de una profesión rentada y reconocida públicamente sino que, además, permitieron el surgimiento de un vasto público lector con intereses y niveles intelectuales muy disímiles. Es decir, un amplísimo conjunto de ciudadanos cuyas necesidades lectoras, tanto en la apropiación como en la múltiple variación de sus prácticas, sería, en muchos momentos 


\section{Susana Romanos de Tiratel / Graciela M. Giunti / Alejandro E. Parada}

de nuestra historia, propicia para el desarrollo sostenido de las revistas literarias.

Dentro del contexto del presente trabajo, resulta imposible señalar un listado de dichas publicaciones. Es importante, empero, señalar algunos de los títulos más importantes. En primera instancia, dos publicaciones anteriores a 1852: LaA bejaA rgentina(1822), publicación mensual de la Sociedad Literaria, con una gran variedad temática que incluía la Literatura; y La M oda (1837-1838), una "gaceta semanal, de música, de poesía, [y] de literatura", en la que intervinieron varios de los hombres vinculados a la generación romántica de 1837. Sin embargo, luego de estos antecedentes, la segunda mitad del siglo XIx inaugurará una cantidad significativa de títulos (aunque en algunos casos lo literario formaba parte de un conglomerado de disciplinas ajenas): El Plata científico y literario (1854) fundado por M iguel Navarro Viola, Revista del Paraná (1861) bajo la dirección de V icente G. Q uesada, y Revista de Buenos Aires (1863) a cargo de los ya citados $N$ avarro $\checkmark$ iola y $Q$ uesada. A las que deben agregarse la Revista del Río dela Plata (1871), N ueva Revista deBuenos Aires (1881), Revista Argentina (1868 y 1880), La N ueva Revista (1893) dirigida por "A níbal Latino", Revista de A mérica (1894) bajo los auspicios de Rubén D arío y Ricardo Jaimes Feryre, La Biblioteca (1896) por Paul G roussac, Juventud (1897), El M ercurio deA mérica (1898), Preludios (1901), I deas (1903), I deas y Figuras (1909), Renacimiento (1909), Verbum (1912), La Cruz del Sur (1913), etc.

No obstante, el siglo xx señala la aparición de las tres revistas literarias argentinas con mayor prestigio y reconocimiento internacional: Nosotros (1907) cuyos directores fueron Roberto F. G iusti y Alfredo A. Bianchi, M artín Fierro (1924), y Sur (1931) dirigida por Victoria 0 campo. La lista es interminable, pues numerosos títulos trascendieron las fronteras y se conocieron en América Latina y España, tales como Inicial, Ideas, A riel, La LiteraturaA rgentina, Ficción, Proa, V aloraciones, O rientación, Sustancia, Vértice, Crisol, Sol y Luna, Libra, M egáfono, etc. (Fernández, 1943; Rojas, 1948; Laf leur, Provenzano y Alonso, 1962).

Esta pequeña y arbitraria selección de publicaciones (que no aborda la extraordinaria cantidad de títulos que aparecieron a partir de la década del cuarenta hasta la actualidad) demuestra la notable riqueza y variedad de las revistas literarias en nuestro país. Es por ello que el estudio de la visibilidad y representación de la moderna literatura periódica en la Argentina se presenta como un hecho fundamental para controlar nuestra producción en esa temática, y para tratar de comprender los actuales criterios de selección e inclusión de las mismas en las bases de datos internacionales.

\section{CONTEXTO TEÓRICO}

La investigación se encuadra dentro de las formulaciones planteadas por M ichael Keresztesi, quien define a las disciplinas como conocimiento que produce y difunde sistemas, donde la investigación surge como la actividad central, pero no puede ocurrir aislada de las fuerzas que influyen sobre la ejecución de la tarea de indagación. La Bibliografía, por lo tanto, debe ver a una disciplina como un sistema de vínculos con muchos niveles donde cada acción productiva y de apoyo engendra los efectos correspondientes en la literatura de la disciplina y en el mecanismo informativo que provee el acceso a ésta. Las interrelaciones entre investigación y bibliografía se vuelven obvias cuando se examinan las disciplinas científicas en una matriz evolutiva. Así, identifica tres estadios en el crecimiento de un área: el estadio pionero, el de elaboración y proliferación y el estadio del establecimiento; y tipifica la producción que caracteriza a cada estadio y las herramientas de acceso a la misma. Además, el autor traza la topografía de las disciplinas científicas cuando identifica sus cuatro dimensiones: epistemológica, sociológica, histórica y bibliográfica (Keresztesi, 1982: 8-13).

Un área primaria de interés en la dimensión bibliográfica son los procesos de investigación, comunicación y difusión del conocimiento junto con el origen, la cantidad y la distribución de la literatura especializada por formatos. El aspecto más crítico es el aparato de la información: cómo satisface las necesidades de la disciplina, la estructura y el nivel de sofisticación del acceso bibliográfico, las capacidades de las herramientas de referencia, el estado del alfabetismo informativo, las consideraciones políticas y de servicio en los planes locales, nacionales e internacionales son algunos de los aspectos clave (Keresztesi, 1982: 23-24).

\section{MÉTODOS Y PROCEDIMIENTOS}

Para recolectar los datos se usaron métodos cuantitativos; las fuentes empleadas son las listas de revistas analizadas en los servicios de indización y resúmenes. Para definir la población e identificar certeramente los títulos se recurrió a los directorios o a las bibliografías de periódicas.

Las técnicas aplicadas pueden responder con exactitud a las preguntas: ¿C uáles son las tasas de representatividad, de visibilidad, de solapamiento y de vacancia de la producción hemerográfica argentina especializada en Filología, Literatura y Lingüística en las bases de datos 
bibliográficos internacionales, tanto pluridisciplinarias como unidisciplinarias?; y ¿Existen tasas diferenciales entre las bases de datos pluridisciplinarias y las unidisciplinarias?

A ntes de pasar a los procedimientos seguidos es necesario formular al gunas precisiones conceptuales, dado que uno de los primeros problemas que se planteó fue el concepto de revista académica, científica, especializada o de investigación. En castellano no existen palabras distintas para denominar a este tipo de periódica y diferenciarlo de las revistas de interés general o de los boletines de noticias. El inglés tiene matices más ricos para este universo; así, discrimina entrejournal, magazine ynewsletter.

Se consultó una obra dedicada a las seriadas que, citando al ALA G lossary of Library and Information Sciencede1983, define journal como una periódica, especialmente una que contiene artículos eruditos y/ o difunde información en curso sobre investigación y desarrollo en un campo temático particular. $\mathrm{M}$ ás adelante, $\mathrm{N}$ isonger enumera las características de las revistas científicas: los autores son expertos que escriben en un estilo académico para una audiencia especializada sobre temas de investigación; suele tener un aspecto físico serio, notas al pie de página y bibliografías, un comité editorial, puede contar con evaluadores externos así como estar cubierta por índices especializados por materias y tiene una circulación y tiradas limitadas ( $\mathrm{N}$ isonger, 1998: 4-5). A esto se puede agregar el tipo de publicador: universidades, asociaciones y colegios profesionales, academias, institutos o centros de investigación y editoriales comerciales dedicadas a la publicación científica.

U na vez aceptado este concepto, se descartaron los boletines de noticias o newsletters, que son seriadas formadas por una o unas pocas hojas impresas que contienen noticias o información de interés, principalmente, para un grupo especializado ( $\mathrm{N}$ isonger, 1998: 6).

Además, se decidió no incluir las series monográficas ni las revistas publicadas después de 1997, porque es altamente improbable que las mismas estén incluidas en las bases de datos internacionales, dado que un principio general para que esto suceda es que la revista demuestre una continuidad en la publicación de, al menos, cuatro años.

La importancia de este recorte previo estribó en la necesidad de tener muy en claro el tipo de revistas que integrarían la lista de periódicas argentinas especializadas en Filología, Literatura y Lingüística para confrontar con las de los servicios de in dización y resúmenes examinados.
0 tros aspectos conceptuales que se han usado en este estudio son los indicadores de representatividad, visibilidad, vacancia y solapamiento los que se han definido así:

- Indicadores de representatividad: es el porcentajedetítulos argentinos en relación con la lista total de títulos analizados en una base de datos.

$$
I R=\frac{\text { títulos de un país en } B \text { ded }}{\text { número total de títulos en } B \text { de } d} \times 100
$$

- Indicadores de visibilidad: es la relación entre la producción total de un país en una temática determinada y el número de títulos indizados en las bases de datos internacionales

$$
\text { IV } i=\frac{\text { títulos indizados en } B \text { de } d}{\text { número total de títulos de la disciplina }} \times 100
$$

- Indicadores de vacancia: es la medida inversa a la visibilidad; determina el porcentaje de título argentinos no analizados en las bases de datos internacionales.

$$
\text { IV a }=\frac{\text { títulos no indizados en } B \text { de d }}{\text { número total de títulos de la disciplina }} \times 100
$$

- Indicadores de solapamiento: mide la cantidad de veces que un mismo título se repite en distintas bases de datos.

Se procedió, en primer término, al establecimiento de una lista de seriadas especializadas en Humanidades y Ciencias Sociales utilizando como fuente el BINPAR (3), compilado por el Centro Argentino de Información Científica y Tecnológica. De ese grupo inicial de cerca de 1.000 títulos se hizo un primer recorte eliminando las series monográficas, las revistas cuyo estado era desconocido, las cesadas y las publicadas después de 1997, llegándose así a una lista al go superior a los 500 títulos. Teniendo en cuenta los criterios previamente establecidos respecto de los publicadores y del tipo de material incluido, se descartaron los boletines de noticias, las revistas de datos estadísticos, de indicadores económicos, bibliográficas o de reseñas y se obtuvo una lista provisional de 356 títulos. Este número se usó para los primeros cálculos, pero dado que algunos criterios debieron flexibilizarse debido a que excluían publicaciones consideradas en las bases de datos, la lista final pasó a conformarse con 400 títulos.

Se diseñó una base de datos en M icrosoft Access donde, para cada título de revista, se establecieron los siguientes campos: título, ISSN, periodicidad, lugar de edición, editor, tipo de entidad editora, fecha, categoría temática, materia 1, materia 2, historia y un campo de control. 
U na vez cargados los datos se pudo recuperar el subconjunto Filología, Literatura y Lingüística con 44 títulos lo que representa un $11 \%$ del conjunto $\mathrm{H}$ umanidades y Ciencias Sociales. Para una mayor precisión hay que establecer el porcentaje de este subconjunto dentro del grupo de las revistas de H umanidades y este es 38,3 .

En segundo lugar, se clasificaron las bases de datos bibliográficos - servicios de índices y resúmenes - en internacionales pluridisciplinarias, latinoamerican as pluridisciplinarias e internacionales unidisciplinarias. Para el caso particular de las disciplinas estudiadas en este trabajo, se incluyeron las siguientes:

a) Internacionales pluridisciplinarias

Francis [Sitio Web]. INIST, CNRS. <U RL: http// www.inist.fr>

IBZ : Internationale Bibliographie der Geistes- und Sozial wissenschaftlichen Zeitschriftenliteratur $=I B Z$ : International Bibliography of Periodical Literaturein theFields of Arts and $\mathrm{H}$ umanities and the Social Sciences. [Sitio W eb]. Dietrich V erlag. $\triangleleft$ RL: http:// www.dietrich-bibliogr.de>

Art and $\mathrm{H}$ umanities Citation Index , Institute for Scientific Information.

b) Latinoamericanas pluridisciplinarias

HAPI O nline. [Sitio W eb]. Produced by an editorial staff at the U CLA Latin American Center. <U RL: http:// gseis.ucla.edu/hapi/html>.

c) Internacionales unidisciplinarias

Modern Language Association (M LA) International Bibliographical of Books and Articles on the Modern Languages and Literatures. <U RL: http://mla.org>.

Linguistic and Language Behavior Abstracts (LLBA). « RL: http:// www.csa.com/ csa/ factsheets/ llba.shtml>

Bibliographie linguistique = Linguistic bibliography . «U RL: http:// http:// www.ciplnet.com/ >

\section{ANÁLISIS DE DATOS}

Como se ha dicho precedentemente, se identificaron 44 títulos de revistas argentinas dedicadas a Filología, Literatura y Lingüística. Para determinar su tasa de representatividad, de visibilidad, de vacancia y de solapamiento se confrontaron con las listas de revistas cubiertas por las bases de datos pluridisciplinarias internacionales y latinoamericanas y, finalmente, con las unidisciplinarias.

\section{B ases de datos pluridisciplinarias}

\section{a) Dealcance internacional}

En primera instancia, para identificar el grado de presencia de revistas argentinas en importantes bases de datos internacionales de carácter multidisciplinario, se estudiaron las siguientes: Francis, compilado por del Institut de I'Information Scientifique et Technique (IN IST) e IBZ: InternationaleBibliographieder G eistes-und Sozial wissenschaftlichen Zeitschriftenliteratur= International Bibliography of Periodical Literaturein theFields of Arts and Humanities and the Social Sciences.

Según Romanos de Tiratel, Francis indexa 2.033 periódicas y la Argentina está representada con 2 revistas $(0,1 \%)$. $\mathrm{N}$ inguno de los dos títulos corresponde al área estudiada en este trabajo. U na situación similar ocurre con IBZ, pues dentro de los 4.650 títulos que indiza, sólo 4 son de Argentina y dentro de ellos no incorpora revistas argentinas de Filología, Literatura y Lingüística (Roman os de Tiratel 2002).

Por último, otra importante base de datos internacional, Arts \& H umanities Citation Index del Institutefor Scientific Information (ISI), no incluye ninguna periódica argentina dentro de un universo total de 1.126 títulos (Romanos de Tiratel 2002).

\section{b) Dealcancelatinoamericano}

"El H ispanic A merican Periodicals I ndex es una base dedatos donde la mayoría de la indización inicial está hecha por bibliotecarios y profesores latinoamericanistas que trabajan voluntariamente. Se publica desde 1970. Sólo analiza revistas y lo hace en profundidad. La base presenta una sola lista de periódicas donde incluye tanto los títulos que indexó en el pasado como los que analiza actualmente. Se trabajó con la lista en curso que incluye 228 ítem. América Latina está representada con 135 títulos (59\%) y, dentro de este grupo, la A rgentina alcanza un 4\% (9 periódicas)" (Romanos de Tiratel, 2002). En cuanto a la presencia de revistas literarias argentinas, en ese universo de 9 títulos, sólo una perten ece a nuestra área de interés.

Es importante señalar que no ha sido considerado otro repertorio representativo de este grupo: $\mathrm{H}$ andbook of Latin A merican Studies (HLAS). Esta decisión se debe a su indización excesivamente selectiva de las revistas incluidas, lo que llevaría a una distorsión de nuestros resultados porque la lista de títulos seleccionados no siempre se ve representada con artículos indizados en dicha base. 


\section{Bases de datos unidisciplinarias}

Se identificaron tres bases de datos internacionales que cubren las áreas temáticas estudiadas: M odern Language Association (M LA) International Bibliographical of Books and Articles on the Modern Languages and Literatures; Linguistic and LanguageB ehaviour A bstracts (LLBA); y Bibliographie linguistique = Linguistic bibliography.

Al cotejar los 44 títulos de Filología, Literatura y Lingüística con las listas de revistas indizadas por las bases antes mencionadas se obtuvo como resultado un total de 17 títulos argentinos representados, es decir, una visibilidad de un $38,6 \%$ y una vacancia del $61,4 \%$, lo que marca en el último caso una seria dificultad para acceder a los contenidos de las revistas (A nexo I).

M LA International Bibliographyesuna base de datos que indiza temáticamente tanto libros como artículos de publicaciones periódicas referidos a Literaturas Modernas, Filología, Lingüística y Folklore. La indización está realizada por bibliógrafos norteameri-canos y por representantes extranjeros de diferentes países. A nualmente incorporan al rededor de 50.000 registros. Esta base de datos cubre cerca de 7.100 títulos, de los cuales sólo 4.400 son indexados en la actualidad. Tomando este último valor como referencia, la presencia de publicaciones argentinas es de 12 títulos, o sea, una tasa de representatividad del $0,3 \%$ y $27,2 \%$ de visibilidad (cabe aclarar que uno de ellos no posee ISSN y, por lo tanto, no está registrado en BINPAR).

Respecto del indicador de representatividad, la Argentina no es una excepción si se compara con el resto de los países de América Latina, tal como se puede ver na tabla 1.

Linguistics \& Language Behavior A bstracts (LLBA) comenzó a publicarse en 1973 y, actualmente, su edición está a cargo de Cambridge Scientific Abstracts. Este repertorio contiene alrededor de 293.200 citas de Filología y Lingüística, e incorpora 15.000 registros por año que corresponden a 1.246 periódicas. Las publicaciones incluidas tienen distintos niveles de cobertura: indizadas totalmente, indizadas en más de un $50 \%$ y, por último, aquéllas que corresponden a menos del $50 \%$.
Dentro de este conjunto se han detectado cinco títulos de revistas argentinas, lo que implica una representatividad del $0,4 \%$; sin embargo, sólo dos títulos se especializan en Filología y Lingüística: una es analizada exhaustivamente y la restante en forma parcial. Por otra parte, es necesario señalar que las otras tres publicaciones son de temática general $y$, de este modo, han sido consideradas "selective sources": se indiza menos del $50 \%$ de su contenido; por consiguiente, no fueron tenidas en cuenta en los cálculos. En consecuencia, el repertorio LLBA sólo considera dos revistas de Filología/ Lingüística cuando en la Argentina existen 16 títulos de estas disciplinas; por lo tanto, la visibilidad es del $12,5 \%$.

U na vez más, los porcentajes de representatividad de los diferentes países de A mérica Latina demuestran que la Argentina no es un caso aislado; así se observa en la tabla 2. 
Por su parte, la Bibliographie linguistique o Linguistic bibliography, compilada bajo la responsabilidad del Comité international permanent de linguistes (CIPL) es reconocida internacionalmente como un trabajo masivo de documentación. Cubre Lingüística general y disciplinas relacionadas (Psicología, Sociología, Antropología y Ciencia de la Computación), y lenguas y dialectos individuales de todo el mundo e identifica libros, reseñas y artículos de muy diversos países desde 1939 hasta la fecha. Todas las publicaciones se registran en su lengua original. La cobertura se limita a dos aspectos: sólo incluye publicaciones científicas y excluye folletos populares y trabajos pedagógicos (si bien la restricción pedagógica se modera en el caso de lenguas menos conocidas); y solo se listan publicaciones de Lingüística aplicada si tienen implicaciones teóricas. Indiza 2.441 títulos, cinco son argentinos, lo que implica una tasa de representatividad del $0,2 \%$ y de visibilidad del $25 \%$. Cabe aclarar que este último porcentaje toma en cuenta cuatro títulos dado que el que se descarta no es específico de Lingüística y por tanto no forma parte del subconjunto de los 16 títulos dedicados a la Filologíal Lingüística.

Por fin, la representatividad de títulos latinoamericanos en la Bibliographie Linguistique vuelve a marcar índices similares a los de las dos bases anteriores, confirmándose así que el caso argentino se encuentra dentro de una problemática mucho más amplia (tabla 3).

0 tro elemento de interés es que únicamente dos títulos están presentes en dos de las bases estudiadas: M LA y LLBA. N o obstante, este dato posee cierta relatividad ya que M LA incluye, además, Literatura.

Como ejemplo ilustrativo, y con la finalidad de observar estos datos desde otro ángulo, se estudió una bibliografía de narrativa argentina para el período 1970-2002 (Parada, Giunti, Romanos de Tiratel). El análisis dio los siguientes resultados: la revista argentina más citada (Revista de Literaturas M odernas, M endoza) no ha sido seleccionada por M LA, mientras que los otros cuatro títulos, en orden de importancia, si lo están. Esta aparente paradoja se explica porque los compiladores de la bibliografía consultaron el catálogo de la Biblioteca de la A cademia A rgentina de Letras, institución que ha hecho la catalogación analítica de la revista mencionada. (A nexo II)

\section{DISC USIÓN}

Retomando la supuesta contradicción explicada en el párrafo anterior, es conveniente afirmar que la misma avala nuestra posición porque demuestra que cuando se hace una investigación sobre un tema argentino y se recurre a fuentes locales que registran la producción del país se logra una recuperación significativa que, tal como se evidencia en este caso, no se obtiene consultando solamente bases internacionales.

Esta investigación, hasta el momento, ha podido determinar los indicadores de representatividad, de visibilidad y de vacancia de las publicaciones periódicas en Filología, Literatura y Lingüística, sin embargo, los mismos no pueden explicar los motivos por los cuales los centros internacionales de indización eligen ciertos títulos y no otros, dado que no se ha logrado identificar patrones regulares al respecto.

Por ejemplo, si consideramos las instituciones editoras del conjunto de 44 revistas argentinas para estas áreas, encontramos que 34 son publicadas por universidades 0 institutos de investigación y las diez restantes por entidades privadas, sin embargo, en relación con el subconjunto de 17 títulos indizados en bases internacionales unidisciplinarias once son de universidades 0 institutos de investigación y seis de entidades privadas. Un patrón ampliamente aceptado en los medios académicos es que las revistas institucionales tienen más chances de ser reconocidas fuera del país, sin embargo, los guarismos presentados no revelan esa tendencia dado que, en términos absolutos, las bases de datos indizan por igual uno (11:17) y otro tipo de periódica (6:17), mientras que, en términos relativos, las revistas emanadas de entidades privadas tienen más visibilidad que las institucionales (6:10 contra 11:34). 


\section{Las revistas argentinas de filología, literatura y lingüística: visibilidad en bases de datos internacionales}

Si consideramos la regularidad como factor determinante para ser incluido en las bases de datos internacionales, en el caso de las disciplinas estudiadas pareciera no tener, prácticamente, incidencia alguna. Lo mismo sucede con el tan mentado sistema de arbitraje (mal denominado de referato) porque un número importante de las revistas indizadas no declara tenerlo. Así, queda demostrada la dificultad intrínseca del estudio de las publicaciones periódicas, porque la historia de cada uno de los títulos entraña en sí misma una serie de factores, tan to aleatorios como previsibles. Por lo tanto, este tipo deinvestigaciones constituye, a veces, un área de difícil asedio.

Por otra parte, es una ley de la compilación bibliográfica que a mayor cobertura geográfica o temática se corresponde un nivel más alto de selectividad y la tendencia imperante se inclina hacia la exhaustividad respecto de lo producido en el país que elabora la base de datos, tal como lo hacen los países centrales. De modo tal que únicamente vemos lo que se nos muestra y esto constituye sólo una ínfima parte de lo publicado por nuestros especialistas. No obstante, tal como se ha afirmado, no se puede responsabilizar de esta situación a otros sino a las propias estructuras institucionales de cada país, únicas responsables del control bibliográfico de las distintas disciplinas, ya sea en conjunto 0 individualmente.

Se percibe en los países periféricos una tensión dialéctica entre el reconocimiento internacional y el reconocimiento local que, en el estadio actual de desarrollo de las disciplinas, no ha podido sintetizarse satisfactoriamente. Hasta que no se resuelva quedarán, al menos, dos áreas problemáticas sin solucionar: la compilación de la bibliografía nacional especializada y la planificación de políticas científicas consecuentes que sepan determinar las necesidades diferenciales entre las disciplinas y logren así incorporarlas a la corriente mundial.

\section{REFEREN CIAS}

ARMENDÁRIZ SÁNCHEZ, Saúl; ORDONEZ ALONSO, M. $M$ agdalena. Las revistas académicas de $\mathrm{H}$ istoria en $\mathrm{H}$ ispanoamérica: un punto de vista. En Clio, n. 3, 1998. Disponível em: <http:// clio.rediris.es/articulos/las_revistas_academicas_de_histo.htm>. Consulta: 22 maio 2003.

CETTO, Ana María; KAI-INGE, Hillerud. (Comp.). Publicaciones científicas en América Latina. París : International Council of Scientific Unions, 1995. $305 \mathrm{p}$.

; A LONSO, O ctavio. (Comp.). Publicaciones científicas en A mérica Latina. París : International Council of Scientific Unions, 1999. $489 p$

DAY, R. A. Scientific journals: an endangered species. International Forum of Information and Documentation, v. 1, n. 3, p. 6-8, 1976.
FERN AN DEZ, Juan Rómulo. Historia del periodismo argentino. Buenos Aires : Perlado, 1943. p. 107-112.

FRAZIER, Ken. SPARC: encouraging new models of disseminating knowledge. Collection Building, v. 19, n. 3, p. 117-123, 2000.

FU EN ;MAYOR, P.; ABDEL, M. Investigar y publicar. Interciencia, v. 20, n. 1, p. 40-46, 1995. Disponível em: <http:// www.interciencia.org/v20_01/ ensayo02.htm>.

TARGIN O, M aria das Graças; GARCIA, Joana Coeli Ribeiro. Ciência brasileira na base de dados do Institute for Scientific Information (ISI). Ciência da Informação, Brasília, v. 29, n. 1, p. 103-117, 2000.

HAU PT, Cecilia. La 8a Reunión sobre las Revistas Académicas y de Investigación. Las revistas científicas latinoamericanas: su difusión y acceso a través de bases de datos. Biblioteca U niversitaria. N ueva época, v. 3, n. 2, p. 122-127, 2000. Disponível em: <http:// www.dgbiblio.unam.mx/ servicios/ dgb/ publicdgb/ bole/ fulltext/ vollll2/ octava.pdf>. Acesso em: 23 jun. 2003.

HOUGHTON, Bernard. Scientific periodicals: their historical development, characteristics and control. London : Linnet Books, 1975. $135 \mathrm{p}$.

KERESZTESO, Michael. The science of bibliography: theoretical implications for bibliographic instruction. OBERMAN, Cerise; STRAU CH, Katina. (Ed.). Theories of bibliographic education. N ew York Bowker, 1982. p. 1-26.

KRAUSKOPF, M anuel; VERA, M aría Inés. Las revistas latinoamericanas de corriente principal: indicadores y estrategias para su consolidación. Interciencia, v. 20, n. 3, p. 144-148, 1995. Disponível em: 〈http:// www.interciencia.org/v20_03/art05/ index.html>. A cesso em: 23 jun. 2003.

KRONICK, David A. A history of scientific and technical periodicals: the origins and development of the scientific and technical press, 1665-1790. 2. ed. Metuchen, N.J. : Scarecrow, 1976. 336 p.

LAFLEUR, H éctor René; PRO VENZANO, Sergio D.; A LONSO, Fernando Pedro. Las revistas literarias argentinas (1893-1960). Buenos Aires : Ediciones Culturales Argentinas, 1962. 282 p.

LICEA DE ARENAS, Judith. M edios y mensajes de la ciencia: la revista científica. Ciencias de la información, v. 25, n. 1, p. 2-12, 1994.

MORRIS, Sally. Learned journals and the communication of research. Learned Publishing, v. 11, n. 4, p. 253-258, 1998.

NISO N GER, Thomas E. 1998. M anagement of serials in libraries. Englewood, Colo. : Libraries U nlimited, 1998. 433 p.

PARADA, Alejandro E.; GIUNTI, Graciela M.; TIRATEL, Susana Romanos de. Bibliografía de la narrativa argentina contemporánea (1970-2002). [S. I. : S. n.], 2003.

PITERNICK, Anne B. Attempts to find alternatives to the scientific journal: a brief review. Journal of A cademic Librarianship, v. 15, n. 5, p. 260-266, 1989.

PU CCIO, Joseph A. Serials reference work. Englewood, Colo. : Libraries U nlimited, 1989. 228 p.

RATTO DE SALA, M aría Cristina; DELLAMEA, A malia Beatriz. Difusión, acceso y visibilidad de publicaciones científicas seriadas de Iberoamérica. El sistema Latindex. Dominguezia, v. 17, n. 1, 2001. Disponível em: <http:// dominguezia.org.ar/volumen/articulos/175.pdf>. A cesso em: 23 jun. 2003.

ROJAS, Ricardo. Historia de la literatura argentina: ensayo filosófico sobre la evolución de la cultura en el Plata: cuarta parte: Los modernos II. Buenos Aires: Losada, 1948. 901 p. 


\section{Susana Romanos de Tiratel / Graciela M. Giunti / Alejandro E. Parada}

ROMANOS DE TIRATEL, Susana. Acceso a la producción argentina en humanidades y ciencias sociales: representatividad en bases de datos internacionales. CONGRESO INTERNCIONAL DE INFORMECION, 2002, La Habana, Cuba. SEMINARIO INTERNACIONAL DE ESTIDIOS CUANTITATIVOS Y CUALITATIVOS DE LA CIENCIA Y LA TECNOLOGIA. Actas. La Habana : Instituto de Información Científica y Tecnológica. 1 disco óptico.

Guía de fuentes de información especializadas: humanidades y ciencias sociales. 2. ed. Buenos Aires : GREBYD, 2000. $281 \mathrm{p}$.
TALLER DE OTENCION DE INDICADORES BIBLIOMETRICOS Y ACTIVIDAD CIENTIFICA, 2., 2000, M adrid. Relatorías. [S. I. : s. n. ], 2000. <http:// www.ricyt.edu.ar/ Biblioteca/ D ocumentos/ 2brelatorias.pdf>. Acesso em: 23 jun. 2003.

2003, M adrid. Disponível em: <http:// www.ricyt.edu.ar> Acesso em: 23 jun. 2003. 
ANEXO I

Listado de revistas argentinas especializadas en Literatura, Ligüística y Filología

\begin{tabular}{|c|c|c|}
\hline Revista & ISSN & $\begin{array}{l}\text { INDIZADA EN } \\
\text { Base de datos }\end{array}$ \\
\hline Alfinge : Revista de la Sección de Filología & Sin ISSN & BL \\
\hline Anales de Filología clásica & $0325-1721$ & \\
\hline Anales del Instituto de Lingüística & $0325-3597$ & $\mathrm{BL}$ \\
\hline Anclajes & 0329-3807 & MLA \\
\hline Auster & $1511-0121$ & \\
\hline Beckettiana & $0327-7550$ & MLA \\
\hline BFI,TAR. Boletín de estudios lingüísticos argentinos & $0.328-3089$ & \\
\hline BOEHI. Buletín de estudios hispámicus & $1514-0059$ & \\
\hline Boletín de la $\Lambda$ cademia $\Lambda$ rgentina de Letras & $0001-3757$ & MLA \\
\hline CLLLIIIS & $0328-5766$ & MLA \\
\hline Circe de clásicos y modernos & $1514-3333$ & \\
\hline Cuadernos Angers & $1514-0083$ & \\
\hline Cuadernos de literatura inglesa y norteamericana & $0328-9184$ & MLA \\
\hline Cuadernos del sur & 0070-1769 & $\mathrm{BL}$ \\
\hline F.T.C. & $0327-7089$ & \\
\hline Feminaria & $1666-2792$ & MLA-HAPI \\
\hline Filología & $0071-495 X$ & MT $\Lambda$-T. B $\Lambda$ \\
\hline Incipit & $0326-0941$ & MLA \\
\hline Inter Litteras & $0328-8935$ & \\
\hline La gramática generativa. Cuadernos & $0328-624 X$ & \\
\hline Lenguaje en contexto & $0327-067 x$ & BL \\
\hline Letras & $0326-3363$ & \\
\hline Letras de Buenos Aires & 0326-2928 & MLA \\
\hline Lingüística en el aula & $1514-0202$ & \\
\hline Menú de lecturas & $0327-0394$ & \\
\hline ()rbis 'l'ertius & $(0328-8188$ & \\
\hline Piedra libre & $0327-0777$ & \\
\hline Piedra y canto & 0328-1094 & \\
\hline Punto de partida & $0327-8018$ & \\
\hline Punto de vista & $0326-3061$ & MLA \\
\hline Raíces & (0)328-5.375 & \\
\hline Revista argentina de lingüística & $0326-6400$ & MLA- LLBA \\
\hline Revista de estudios clásicos & $0325-3465$ & \\
\hline Revista de estudios de egiptología & $0327-3822$ & $\mathrm{BI}$, \\
\hline Revista de la Saciedad Argentina de Lingüística & $0327-8794$ & \\
\hline Revista de lengua y literatura & 0327-1951 & \\
\hline Revista de letras & $0328-8889$ & \\
\hline Revista de literaturas modernas & $0556-6134$ & \\
\hline Revista universitaria de letras & $0326-1166$ & \\
\hline $\begin{array}{l}\text { RILL. Revista del Instituto de Investigaciones } \\
\text { Linguísticas y Literarias I Iispanoamericanas }\end{array}$ & $0328-1922$ & \\
\hline Signo \& seña & $0.327-8956$ & \\
\hline Stylos & $0327-8859$ & \\
\hline Tcatro XXI & $0328-9230$ & \\
\hline Tokonoma & Sin ISSN & MLA \\
\hline
\end{tabular}


ANEXO II

Bibliografía de narrativa argentina (1970-2002)

Listado de revistas argentinas con su frecuencia de citas

\begin{tabular}{|c|c|c|}
\hline Título de la Revista & $\begin{array}{c}\text { Cantidad de } \\
\text { citas }\end{array}$ & Binpar \\
\hline Argentina en su literatura & 3 & No \\
\hline Boletín de la Academia Argentina de Letras & $* 5$ & $\mathrm{Si}$ \\
\hline Bolelín de Literatura Comparada & 1 & No \\
\hline Bolelín G.E.C. & 1 & No \\
\hline Confluencia & 2 & $\mathrm{Si}$ \\
\hline Crisis & 1 & $\mathrm{Si}$ \\
\hline Cuadernos Angers I a Plata & 2 & $\mathrm{Si}$ \\
\hline Cuadernos de I iteratura & 2 & $\mathrm{Si}$ \\
\hline Cuadernos Tucumanos de Cultura & 1 & $\mathrm{Si}$ \\
\hline F.T.C. & 1 & $\mathrm{Si}$ \\
\hline F1 matadero & 1 & $\mathrm{Si}$ \\
\hline El ojo mocho & 1 & No \\
\hline Fscribas & 2 & $\mathrm{Si}$ \\
\hline Fspacios de crítica y producción & 4 & $\mathrm{Si}$ \\
\hline Feminaria & $* 5$ & Si \\
\hline I etras (UCA) & 1 & $\mathrm{Si}$ \\
\hline I.etras de Buenos Aires & 3 & $\mathrm{Si}$ \\
\hline Lucanor & 2 & $\mathrm{Si}$ \\
\hline Mora & 1 & $\mathrm{Si}$ \\
\hline Mundi & 1 & $\mathrm{Si}$ \\
\hline Orbis Tertius & 2 & $\mathrm{Si}$ \\
\hline Piedra y Canto & $* 5$ & $\mathrm{Si}$ \\
\hline Punto de vista & 6 & $\mathrm{Si}$ \\
\hline Puro cuento & 2 & $\mathrm{Si}$ \\
\hline Repertorio Iatinoamericano & 1 & No \\
\hline Revista de Lengua y Literatura & 1 & $\mathrm{Si}$ \\
\hline Revista de Literaturas Modernas & $* 14$ & Si \\
\hline Silabario & 2 & $\mathrm{Si}$ \\
\hline Sur & 1 & $\mathrm{Si}$ \\
\hline Todo es historia & 2 & Si \\
\hline Tramas para leer la literatura argentina & 1 & $\mathrm{Si}$ \\
\hline Voces entrerrianas & 1 & No \\
\hline
\end{tabular}

La bibliografía de narrativa argentina (1970-2002) registra 606 citas bibliográficas, 78 son de artículos editados en revistas argentinas que representan los 32 títulos de la tabla (26 de ellos están presentes en BIN PAR).

Los títulos en negrita y con asterisco $(*)$ indican los que tienen mayor frecuencia de citas. 